\title{
A General Model and Analysis of Physical Layer Capture in 802.11 Networks
}

\author{
Hoon Chang \\ Computer Science Department \\ Columbia University \\ New York, NY 10027 \\ Email: hchang@cs.columbia.edu
}

\author{
Vishal Misra \\ Computer Science Department \\ Columbia University \\ New York, NY 10027 \\ Email: misra@cs.columbia.edu
}

\author{
Dan Rubenstein \\ Electrical Engineering Department \\ Columbia University \\ New York, NY 10027 \\ Email: danr@ee.columbia.edu
}

\begin{abstract}
While packet capture has been observed in real implementations of 802.11 devices, there is a lack of accurate models that describe the phenomenon. We present a general analytical model and an iterative method that predicts error probabilities and throughputs of packet transmissions with multiple senderreceiver pairs. Our model offers a more accurate prediction than previous work by taking into account the cumulative strength of interference signals and using the BER model to convert a signal to interference and noise ratio value to a bit error probability. This permits the analysis of packet reception at any transmission rate with interference from neighbors at any set of locations. We also prove that our iterative method converges, and we verify the accuracy of our model through simulations in Qualnet. Last, we present a rate assignment algorithm to reduce the average delay as an application of our analysis.
\end{abstract}

\section{INTRODUCTION}

802.11 networks have become so pervasive that it is not uncommon for individual users to install and use their own access points such that the different users' transmissions collide and interfere with one another. However, even with the existing interference, when a client is within a few meters of its access point, transmissions can still be received through a collision. This phenomenon is called physical layer capture [1].

Most research on physical layer capture has focused only on infrastructure networks where many client nodes transmit to one access point [2], [3], [4], [5]. Moreover, these papers consider simplified models of 802.11 DCF operations. Only [6] and [7] provide a model for networks with multiple senders and receivers running a collision avoidance protocol such as 802.11 DCF. However, these works assume that only transmissions from neighboring nodes within a circular area can collide, and that all transmissions involved in a collision completely fail. However, the authors of [8] point out that the cumulative strength of interference affect the absolute performance of a protocol.

In this paper, we provide an analytical model to describe the general effect of interference, including packet capture. Our model considers the cumulative strength of interference

This material was supported in part by the National Science Foundation under CAREER Award No. CNS-560153 and by support from Samsung, the Intel Information Technology Research Council, and Microsoft Research. Any opinions, findings, and conclusions or recommendations expressed in this material are those of the authors and do not necessarily reflect the views of the National Science Foundation. signals from all neighbors and can be extended to model other physical layer effects such as shadowing and multipath fading. We also present an iterative method to compute and solve the complex equations in the model, and prove that our method converges. We then verify the accuracy of these results through simulation. Last, we present a rate assignment algorithm as an application of our model and analysis. The algorithm assigns transmission rates to all senders to reduce the average delivery time of the network.

The rest of our paper is structured as follows: section II reviews related work. Section III describes packet capture in 802.11. Section IV presents our modeling and analysis of interference. Section V presents simulation results with the network simulator Qualnet to support the analysis in the previous section. In Section VI, we present our algorithm to reduce expected delays, and conclude in section VII.

\section{RELATED WORK}

In [9] and [10], the authors perform real experiments with 802.11 implementations that demonstrate the effect of packet capturing. In [9], the authors empirically show that physical layer capture in $802.11 \mathrm{~b}$ occurs more frequently than was previously accounted for in simulation. They identify that frames with strong signal power can be captured even when they arrive later than a signal being received. They investigate existing $802.11 \mathrm{~b}$ simulators, $\mathrm{ns}-2$ and Qualnet, which assume that packet capturing occurs only if the stronger frame arrives first at the receiver, and observe that in reality, throughput unfairness can be significantly (up to $15 \%$ ) higher than what is assumed these simulators. [10] presents empirical results for 802.11 ad hoc networks that indicate that the packet capture may have a significant effect on the performance of an ad hoc 802.11 network despite the use of the RTS-CTSDATA-ACK 4-way handshake.

In [2], the authors present capture probabilities under a variety of capturing assumptions. Here, experiments on an infrastructure network evaluate the system performance in the presence of multipath fading. [3] investigates the capture effects for a wireless local area network (LAN) system in the presence of multipath, shadowing, and near-far effects. [4] also analyzes the effect of capturing over theoretical throughput and delay of a traffic-saturated $802.11 \mathrm{~b}$ basic service area in 
Infrastructure and Ad hoc configurations. In another paper [5], the authors estimate the influence that the capture effect has on the capacity of IEEE $802.11 \mathrm{~b}$ DCF within a single picocell. However, all works except for [4] analyze only transmissions from client nodes to one access point, which is located in the center of a single cell. Even though [4] considers nodes operating in ad-hoc mode, all of [3], [4], [5] simply assume that all senders schedule their transmissions at each slot with the same probability. If each transmission experiences errors with different probabilities, the assumption is invalid. [2] does not take MAC operations into consideration.

Traditional 802.11 DCF analysis such as [11], [12] use the same assumption that the error probability of a transmission is the constant and equal for all nodes. This assumption cannot handle packet captures at differing sender-receiver distances. [13] provides one of the first analytical models of the IEEE 802.11 DCF where both capture and hidden terminals are considered. However, the authors draw a virtual circle around a sender and a receiver and when any nodes transmit at the same time as the sender, the packet from the sender is lost. This assumption also carries over to [6] and [7]. In [6], transmissions are assumed to be corrupt if any nodes within the carrier-sensing range of the sender or the receiver start sending packets during the transmissions. With this assumption, analysis is simpler but cannot account for packet capturing ([6]) or the effect of multiple interfering nodes ([6], [7]). Moreover, analysis techniques in [6] do not compute throughputs if there are more than 10 neighbors around one node (i.e., when $a \geq(n-1)^{-1}$ where $a$ is 0.1107 for 802.11a and $n$ is the number of neighbors).

\section{PACKet CAPTURE IN 802.11}

There are numerous scenarios in which clients are very close to their 802.11 wireless access points, such as in office and residential units. Since signals from neighboring transmissions are often weaker at the receiving end than the primary, desired transmission, some transmitted packets can be "captured", even through collisions. Figure 1 shows simulation results that plot the cumulative throughput of sender-receiver pairs on the $y$-axis as the number of these pairs is varied along the $x$-axis for several transmission rates. $x$ Senders are positioned in the center of $x$ rooms in an area $200 \times 200$ square meters. Each sender's Receiver is placed randomly at a distance 10 meters from the sender. Detailed simulation parameters are found in section V. With this node placement, we perform a simulation in Qualnet and measure total throughputs varying physical layer transmission rates. The results show that transmitting at low speeds performs better due to the capture effect as the number of pairs

In Figure 1, transmitting at lower rates yields higher throughput as the number of nodes increases. This is because packets are captured with lower probability for high transmission rates. When a sender-receiver pair has only a small number of neighbors, packets transmitted at low rates are not lost (i.e., $100 \%$ of packets are captured at receivers) and throughput linearly increases with the number of nodes. As

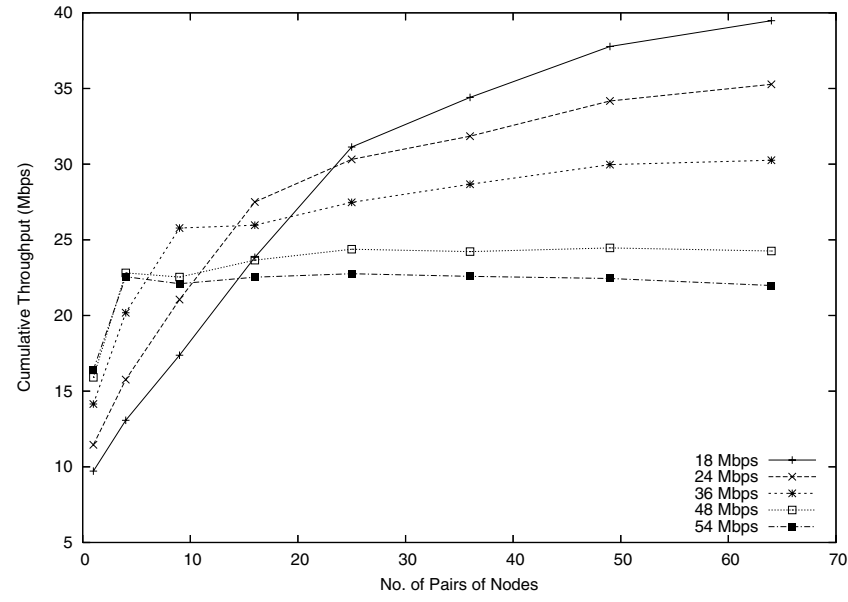

Fig. 1. The effect of packet capturing on cumulative throughput.

the number of neighbors is increased, packet loss rates escalate and throughput reduces as a result.

\section{NumERICAL ANALYSIS}

\section{A. System Throughput}

In the analysis, we assume that each sender always has packets to send (i.e., is saturated) and transmits at a fixed rate in the range of $r_{1}$ to $r_{s}$. Note that different senders can have different transmission rates. Furthermore, we assume that senders are sufficiently close to their receivers so that they do not experience any packet losses in the absence of any collisions.

We use Bianchi's approximation [11] in our model: each packet is lost with a constant and independent probability regardless of the number of retransmissions attempted. However, unlike [11], we do not assume the probability must be the same for all senders as in. Each sender has its own error probability, which depends on the activities of the other senders and the channel condition. Let $p_{i}$ denote the error probability for node $i$. If $p_{i}$ is equal to zero, packets from node $i$ are completely captured by its receiver, which is not modeled by Bianchi's work. Similar work with different error probabilities is found in [14].

Given the error probability, MAC protocols can be viewed as dynamic systems whose feedback information is the probability $p_{i}$ and the corresponding output is the transmission probability $\tau_{i}$ at each slot [6]. Under saturated conditions, $\tau_{i}$ can be obtained for 802.11 DCF via the Markov model in [11].

$$
\tau_{i}=\frac{2\left(1-2 p_{i}\right)}{\left(1-2 p_{i}\right)\left(W_{0}+1\right)+p_{i} W_{0}\left(1-\left(2 p_{i}\right)^{m}\right)},
$$

where $W_{0}$ is the minimum contention window size and $m$ is the value such that the maximum contention window size is equal to $2^{m} W_{0}$.

For simplicity, we derive a linear equation to estimate $\tau_{i}$. This is done by calculating values of $\tau_{i}$ using Equation 1, varying $p_{i}$ from 0 to 0.7 at increments of 0.05 and applying the least squares method to the sample data. As a result, we 
approximate $\tau_{i}$ as $\max \left(\beta-\alpha p_{i}, 0\right)$. Applying 802.11a operating parameters, we calculate that $\alpha$ to be 0.180820691 and $\beta$ is 0.128201376 with the coefficient of determination equal to 0.991393081 . Note that the coefficient of determination is an indicator of how well the linear equation fits. It ranges in value from 0 to 1 and the coefficient 0.991393081 indicates that there is almost a perfect correlation in the estimated and actual value of $\tau_{i}$.

Next, we consider the system throughput. We assume that all nodes are within a single carrier-sensing range; each node can sense signals from all other nodes and nodes do not need to exchange RTS/CTS messages to reserve the channel. Since each node independently schedules its transmission, the probability that no senders start transmissions in a given slot is $\prod_{i}\left(1-\tau_{i}\right)$. If any node begins a transmission at the slot, the channel is occupied during the transmission time and a new backoff slot begins after time DIFS from the transmission. Let $P_{T X}(A)$ be the transmission probability of a node set $A$, which equals $1-\prod_{i \in A}\left(1-\tau_{i}\right)$. The average interval time $T_{S}$ between two adjacent backoff slots is given by:

$$
\begin{aligned}
T_{S}= & T_{S L O T}\left(1-P_{T X}(N)\right)+ \\
& \left(\overline{T_{T X}}+T_{D I F S}\right) P_{T X}(N),
\end{aligned}
$$

where $T_{S L O T}$ and $T_{D I F S}$ are the slot times, the DIFS duration in 802.11, and $N$ is a set of all senders.

$\overline{T_{T X}}$ denotes the average transmission time. Note that if there is a collision, the total transmission time equals the longest time of transmissions involved in the collision. Let a node set $R_{i}$ include all senders transmitting at rate $r_{i}$, with the rates $r_{i}$ be arranged in ascending order (that is, $r_{i}<r_{j}$ for $1 \leq i<j \leq s)$. Given the transmission probability $P_{T X}\left(R_{i}\right)$, the transmission time $T_{T X}\left(r_{i}\right), \overline{T_{T X}}$ equates to:

$$
\overline{T_{T X}}=\sum_{i=1}^{s} T_{T X}\left(r_{i}\right) \frac{P_{T X}\left(R_{i}\right) \prod_{j<i}\left(1-P_{T X}\left(R_{j}\right)\right)}{P_{T X}(N)}
$$

$T_{T X}\left(r_{i}\right)=T_{D A T A}\left(r_{i}\right)+T_{S I F S}+T_{A C K}\left(r_{i}\right)+2 \delta$, where $\delta$ is the maximum propagation delay. Note that we include the transmission time of acknowledgment. If a received packet is corrupted, the receiver does not respond to it. However, since close distance between sender and receiver allows many packets to be captured, receivers may reply with acknowledgments at high probability even when collisions occur. Our simulation results in Section $\mathrm{V}$ justify this assumption.

We consider the average number of delivered packets during time $T_{S}$, by assuming that a success of every transmission from node $i$ is independently determined by the error probability $p_{i}$. Given the transmission probability $\tau_{i}$, the average number of delivered packets is:

$$
N_{S}=\sum_{i} \tau_{i}\left(1-p_{i}\right) .
$$

Finally, using $T_{S}$ and $N_{S}$, the total throughput $T$ is given by Equation 5 .

$$
T=\frac{N_{S} \times L}{T_{S}}
$$

where $L$ is the packet length in bits.

So far, we have computed the total throughput from the error probabilities of all nodes. The error probabilities are determined by distances to and the transmission probabilities of interferers. In the next section, we will consider in depth the relationship between the error probabilities and the activity of neighbors.

\section{B. Interference Range}

Although there is a significant body of literature that analyzes 802.11 MAC behavior, most of them are based on Bianchi's work [11], which assumes that all collisions result in packet losses. The assumption is valid only when all senders are close to each other and interference signals are strong enough to corrupt transmissions. In other cases, some packets can be delivered without errors even through their transmissions overlap in terms of time.

Another approach is to draw a virtual circle around a node and consider only nodes with the range as in [6]. That is, if any nodes within the range transmit during a transmission of the center node, the packet from the center node is lost. This is a reasonable assumption if only two senders are involved in a collision. In 802.11, the error probability of a transmission sharply decreases as the signal to interference and noise ratio (SNIR) grows over a certain threshold. Any signal that is weaker than the threshold can be ignored.

If, however, more than two senders are involved in a collision, the cumulative strength of multiple interference signals must be considered. For example, according to simulation results in Qualnet, the SNIR threshold for reception of 1000-byte packets at $54 \mathrm{Mbps}$ without error is $19.68 \mathrm{~dB}$ while complete loss occurs for an SNIR value $17.85 \mathrm{~dB}$. The strength of signals from two senders at the same location is $3 \mathrm{~dB}$ larger than that from a single sender and the cumulative strength makes a significantly changes the error probability at the receiver. Note that the higher the transmission probability $\left(\tau_{i}\right)$, the more often such concurrent transmissions occur. As an example, given 36 senders and assuming the transmission probability is 0.05 for all senders, the probability where two or more neighbors concurrently transmit is $\sum_{i=2}^{36}\left(\begin{array}{c}36 \\ i\end{array}\right) 0.05^{i}(1-0.05)^{36-i}=0.543270694$. The transmission probability 0.05 is value commonly used in our simulation. The concurrent transmission probability, which is more than half, can not be ignored.

Let us define some additional notation that will facilitate the discussion of concurrent transmissions. Let $N$ be a node set and $2^{N}$ be the power set of $N$. Define $T_{i}(J)$ be the probability that, with the possible exception of node $i$, only nodes in the subset $J$ of $N$ schedules their transmissions at a given slot. That is, $T_{i}(J)=\prod_{j \in J} \tau_{j} \prod_{k \in N-(J \cup\{i\})}\left(1-\tau_{k}\right)$. For example, the probability of concurrent transmissions from two nodes $j$ and $k$ is $T_{i}(\{j, k\})=\tau_{j} \tau_{k} \prod_{l \in N-\{i, j, k\}}\left(1-\tau_{l}\right)$. Similarly, we can extend the definition such that $T_{i, m}(J)=$ $\prod_{j \in J} \tau_{j} \prod_{k \in N-(J \cup\{i, m\})}\left(1-\tau_{k}\right)$.

Next, we introduce interference functions. Interference function $f_{r_{i}}(J)$ maps a given interference signal strength from all 
nodes in $J$ to a packet error probability at transmission rate $r_{i}$. In our method, $f_{r_{i}}$ first computes the total signal strength from nodes in a given set $J$ and calculates the signal to noise and interference ratio (SNIR) as in [15]. With the result, it maps the SNIR to a bit error probability using the mapping table we derive from Qualnet, and finally calculates a packet error probability with a given packet size. Other mapping tables based on experimental results can be used.

Note that other effects of the physical channel can be considered in the interference function. If packets are lost with probability $q_{i}$ without interference, a new interference function $f_{r_{i}}^{\prime}(J)=f_{r_{i}}(J)+\left(1-f_{r_{i}}(J)\right) q_{i}$, can be used to account for the channel losses. Shadowing and multipath fading can be reflected in similar ways.

Using this notation, the error probability $p_{i}$ of a transmission from node $i$ is:

$$
p_{i}=\sum_{J \subset 2^{N-\{i\}}} f_{r_{i}}(J) T_{i}(J) .
$$

In order to compute the system throughput, all we need do is solve Equation 6 and $\tau_{i}=\beta-\alpha p_{i}$, which is an approximation of Equation 1. However, Equation 6 is not linear and is hard to solve, especially when the number of nodes is large. Hence, we develop an iteration method, which is presented in the next section.

\section{Iteration Method}

Our iteration method starts with initial error probabilities and interference functions and computes new error probabilities as in Algorithm 1. Each iteration consists of two loops. The first loop computes $\tau_{j}^{k}$ for all node $i$ and the second loop calculates new probabilities $p_{i}^{k}$ from the computed $\tau_{i}^{k}$ in the previous steps. The algorithm repeats all steps $K$ times. Note that $K$ is a constant number and independent of the number of nodes.

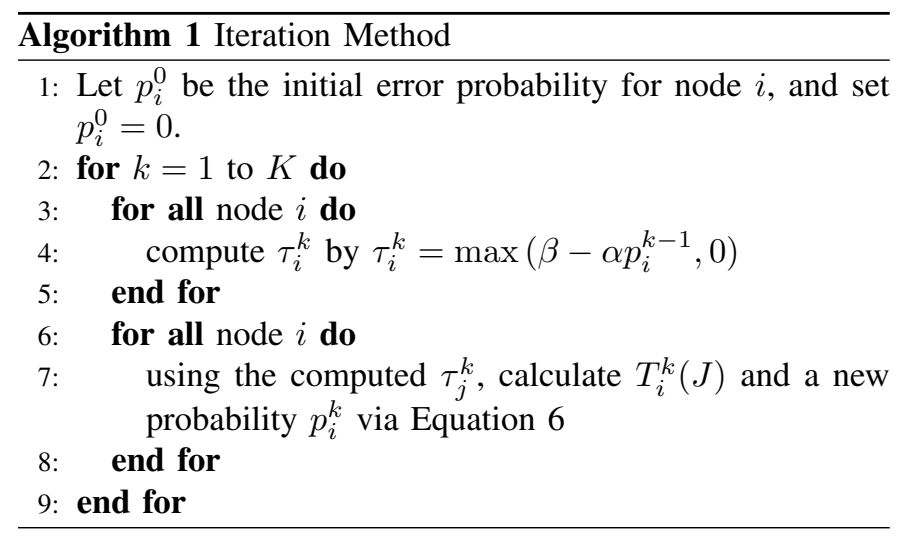

With $n$ nodes, computing $p_{i}^{k}$ at step 7 of Algorithm 1 takes $O\left(n^{n}\right)$ time because $|J|$ can be up to $n-1$ in Equation 6 and calculating $T_{i}^{k}(J)$ takes $O\left(n^{|J|}\right)$. To reduce computation time, we consider only subsets whose size is less than or equal to some value $t$. The approximation of $p_{i}$ with this size limit $t$ is given by Equation 7 .

$$
\widehat{p_{i}}=\sum_{J \subset 2^{N-\{i\}},|J| \leq t} f_{r_{i}}(J) T_{i}(J) .
$$

If subsets with size $t$ or less are taken from the power set, computation time of Equation 6 drops to $O\left(n^{t}\right)$. Repeating the iteration $K$ times increases the total computation time by a constant factor, and thus the total time of our method is $O\left(n^{t}\right)$. Note that the computation proposed in [6] takes $O\left(n^{3}\right)$ in general. In Section V, we show the accuracy of the iterative method as a function of $t$.

Now, we prove the convergence of our method. Let $D_{i}^{k}=$ $\left|p_{i}^{k}-p_{i}^{k-1}\right|$ and $D^{k}=\max _{i}\left(D_{i}^{k}\right)$. The key idea is to show $D^{k}$, the maximum difference between two adjacent iteration steps monotonically decreases. With the property, $p_{i}^{k-1}-D^{k-1}<$ $p_{i}^{k}<p_{i}^{k-1}+D^{k-1}$ and $p_{i}^{k}$ converges into a small range after finite iterations. Four lemmas are introduced first to support our theorem.

Lemma 1: $2\left|\tau_{i}^{k}-\tau_{i}^{k-1}\right|<D_{i}^{k-1}$.

Proof: Since $\tau_{i}^{k}=\beta-\alpha p_{i}^{k-1},\left|\tau_{i}^{k}-\tau_{i}^{k-1}\right|$ is equal to $\alpha\left|p_{i}^{k-1}-p_{i}^{k-2}\right|$, which is $\alpha D_{i}^{k-1}$. Because $\alpha<1 / 2$ in 802.11, $2 \alpha D_{i}^{k-1}$ is less than $D_{i}^{k-1}$. The lemma is proved.

Now we arrive Lemma 2, which shows inequality of $\mid \tau_{m}^{k}-$ $\tau_{m}^{k-1} \mid$.

Lemma 2: If $\left|\tau_{m}^{k}-\tau_{m}^{k-1}\right| \geq\left|\tau_{j}^{k}-\tau_{j}^{k-1}\right|$, then we have that $\left|\tau_{m}^{k}-\tau_{m}^{k-1}\right|$ is greater than or equal to the sum of $\mid \tau_{m}^{k} \tau_{j}^{k}-$ $\tau_{m}^{k-1} \tau_{j}^{k-1} \mid$ and $\left|\tau_{m}^{k}\left(1-\tau_{j}^{k}\right)-\tau_{m}^{k-1}\left(1-\tau_{j}^{k-1}\right)\right|$.

Proof: Let $\tau_{m}^{k}-\tau_{m}^{k-1}$ be $\delta_{m}$ and $\tau_{j}^{k}-\tau_{j}^{k-1}$ be $\delta_{j}$. With $\delta_{m}$ and $\delta_{j}$, the first part is:

$$
\begin{aligned}
\tau_{m}^{k} \tau_{j}^{k}-\tau_{m}^{k-1} \tau_{j}^{k-1} & =\tau_{m}^{k} \tau_{j}^{k}-\left(\tau_{m}^{k}-\delta_{m}\right)\left(\tau_{j}^{k}-\delta_{j}\right) \\
& =\delta_{m} \tau_{j}^{k}+\delta_{j} \tau_{m}^{k}-\delta_{m} \delta_{j} .
\end{aligned}
$$

Now consider the second part.

$$
\begin{aligned}
& \tau_{m}^{k}\left(1-\tau_{j}^{k}\right)-\tau_{m}^{k-1}\left(1-\tau_{j}^{k-1}\right) \\
& \quad=\delta_{m}-\left(\delta_{m} \tau_{j}^{k}+\delta_{j} \tau_{m}^{k}-\delta_{m} \delta_{j}\right) .
\end{aligned}
$$

To use $|A|+|B|=\max (|A-B|,|A+B|)$, we compute sum and difference of the two parts. From Equation 8 and 9, the sum is $\left|\delta_{m}\right|$. The difference is given by $\mid 2\left(\delta_{j} \tau_{m}^{k}+\delta_{m} \tau_{j}^{k}-\right.$ $\left.\left.\delta_{m} \delta_{j}\right)-\delta_{m}\right) \mid$, which is less than or equal to $\mid \delta_{m}\left(2\left(\tau_{m}^{k}+\tau_{j}^{k}-\right.\right.$ $\left.\left.\delta_{j}\right)-1\right) \mid$ because $\left|\delta_{m}\right| \geq\left|\delta_{j}\right|$.

Note that $\tau_{m}^{k}$ and $\tau_{j}^{k}$ is less than or equal to $\beta$ for any $k$. Since $\beta$ is less than $1 / 4$ and $\tau_{j}-\delta_{j} \leq \tau_{j}$, we have that $\tau_{m}^{k}+\tau_{j}^{k}-\delta_{j}<1 / 2$. Thus, the difference becomes less than $\left|\delta_{m}\right|$. We have shown that both of the sum and the difference is less than or equal to $\left|\delta_{m}\right|$ and the lemma is proved.

We introduce the following notations and Lemma 3.

Definition 1: Let $\Delta T_{i, m}^{k}(J)=\tau_{m}^{k} T_{i, m}^{k}(J)-\tau_{m}^{k-1} T_{i, m}^{k-1}(J)$. With three variables $i, m, j$, define $\Delta T_{i, m, j}^{k}(J)$ such that $\Delta T_{i, m, j}^{k}(J)=\tau_{m}^{k} \tau_{j}^{k} T_{i, m, j}^{k}(J)-\tau_{m}^{k-1} \tau_{j}^{k-1} T_{i, m, j}^{k-1}(J)$.

Definition 2: $\Delta T_{i, \bar{m}}^{k}(J)$ (with an inverse of variable $M$ ) denotes the expression $\left(1-\tau_{m}^{k}\right) T_{i, m}^{k}(J)-\left(1-\tau_{m}^{k-1}\right) T_{i, m}^{k-1}(J)$. For three variables, we can similarly define that $\Delta T_{i, m, \bar{j}}^{k}(J)$ denotes $\tau_{m}^{k}\left(1-\tau_{j}^{k}\right) T_{i, m, j}^{k}(J)-\tau_{m}^{k-1}\left(1-\tau_{j}^{k-1}\right) T_{i, m, j}^{k-1}(J)$. 
Lemma 3: Given set $N$, let a set $N^{\prime}$ be $N \cup\{j\}$ and $j \notin N$. If $\tau_{j}^{k}=\tau_{j}^{k-1}=1$, then $\left|\sum_{J \in 2^{N^{\prime}-\{i, m, j\}}} a_{J} \Delta T_{i, m, j}^{k}(J)\right|=$ $\left|\sum_{J \in 2^{N-\{i, m\}}} a_{J} \Delta T_{i, m}^{k}(J)\right|$ for any $a_{J}, 0 \leq a_{J} \leq 1$.

Proof: It is trivial to show that $N^{\prime}-\{i, m, j\}=N-$ $\{i, m\}$. This is because $j$ is not in either $J$ or $N, T_{i, m, j}^{k}(J)=$ $T_{i, m}^{k}(J)$ by definition. Now, we have that:

$$
\begin{aligned}
\Delta T_{i, m, j}^{k}(J) & =\tau_{m}^{k} \tau_{j}^{k} T_{i, m, j}^{k}(J)-\tau_{m}^{k-1} \tau_{j}^{k-1} T_{i, m, j}^{k-1}(J) \\
& =\tau_{m}^{k} T_{i, m, j}^{k}(J)-\tau_{m}^{k-1} T_{i, m, j}^{k-1}(J) \\
& =\tau_{m}^{k} T_{i, m}^{k}(J)-\tau_{m}^{k-1} T_{i, m}^{k-1}(J) \\
& =\Delta T_{i, m}^{k}(J) .
\end{aligned}
$$

Thus, the lemma is proved.

Using the definitions and Lemma 2 and 3, the next lemma shows that the sum of $\Delta T_{i, m}^{k}(J)$ is bounded.

Lemma 4: Consider node set $N$ and nodes $i, m \in N$. Assume that $\left|\tau_{m}^{k}-\tau_{m}^{k-1}\right|$ is greater than or equal to $\left|\tau_{j}^{k}-\tau_{j}^{k-1}\right|$, where $j \in N-\{i, m\}$. For any numbers $a_{J}, 0 \leq a_{J} \leq 1$, $\left|\sum_{J \in 2^{N-\{i, m\}}} a_{J} \Delta T_{i, m}^{k}(J)\right| \leq\left|\tau_{m}^{k}-\tau_{m}^{k-1}\right|$. In addition, we have that $\left|\sum_{J \in 2^{N-\{i, m\}}} a_{J} \Delta T_{i, \bar{m}}^{k}(J)\right| \leq\left|\tau_{m}^{k}-\tau_{m}^{k-1}\right|$.

Proof: Recall that $T_{i, m}^{k}(J)$ is the probability that only nodes in a subset $J$ among $N-\{i, m\}$ transmit at the same slot. When $N=\{i, m\}, T_{i, m}^{k}(\phi)$ is 1 by definition. We prove the lemma by mathematical induction.

First, let $N=\{i, m\} .\left|\sum_{J} a_{J} \Delta T_{i, m}^{k}(J)\right|$ is $\left|a_{\phi} \Delta T_{i, m}^{k}(\phi)\right|$, which becomes $\left|a_{\phi}\left(\tau_{m}^{k}-\tau_{m}^{k-1}\right)\right|$.

Now, assume that the lemma is true for all node sets $N$ with $n-1$ elements. Consider a set $N^{\prime}$ such that it has $n$ nodes and $N^{\prime}=N \cup\{j\}$. We first prove that $\left|\sum_{J \in 2^{N^{\prime}-\{i, m, j\}}} a_{J} \Delta T_{i, m, j}^{k}(J)\right| \leq\left|\tau_{m}^{k} \tau_{j}^{k}-\tau_{m}^{k-1} \tau_{j}^{k-1}\right|$. Assume that $\tau_{j}^{k}=\tau_{j}^{k-1}=1$. From Lemma 3, the left side of the inequality is equal to $\left|\sum_{J \in 2^{N-\{i, m\}}} a_{J} \Delta T_{i, m}^{k}(J)\right|$. Since the lemma is valid for any size $n-1$ sets, the expression is less than or equal to $\left|\tau_{m}^{k}-\tau_{m}^{k-1}\right|$, which is $\left|\tau_{m}^{k} \tau_{j}^{k}-\tau_{m}^{k-1} \tau_{j}^{k-1}\right|$.

Consider $\tau_{j}^{k}, \tau_{j}^{k-1}<1$. Take out $m$ and $j$ from the set $N^{\prime}$ and put in two new nodes $m^{\prime}$ and $j^{\prime}$ such that $\tau_{m^{\prime}}^{k}=$ $\tau_{m}^{k} \tau_{j}^{k}, \tau_{m^{\prime}}^{k-1}=\tau_{m}^{k-1} \tau_{j}^{k-1}$ and $\tau_{j^{\prime}}^{k}=\tau_{j^{\prime}}^{k-1}=1$. With the two new nodes, $\Delta T_{i, m, j}^{k}(J)=\Delta T_{i, m^{\prime} j^{\prime}}^{k}(J)$. We obtain that $\left|\sum_{J} a_{J} \Delta T_{i, m, j}^{k}(J)\right|=\left|\sum_{J} a_{J} \Delta T_{i, m^{\prime}, j^{\prime}}^{k}(J)\right|$. Since the lemma is valid adding such a node $j^{\prime}$ to any size $n-1$ sets, the expression becomes less than or equal to $\left|\tau_{m^{\prime}}^{k}-\tau_{m^{\prime}}^{k-1}\right|$, which is $\left|\tau_{m}^{k} \tau_{j}^{k}-\tau_{m}^{k-1} \tau_{j}^{k-1}\right|$.

In the same way, $\left|\sum_{J \in 2^{N-\{i, m, j\}}} a_{J} \Delta T_{i, m, \bar{j}}^{k}(J)\right|$ is proved to be less than or equal to $\left|\tau_{m}^{k}\left(1-\tau_{j}^{k}\right)-\tau_{m}^{k-1}\left(1-\tau_{j}^{k-1}\right)\right|$. Note that two inequalities we have are true for any numbers $a_{J}$ in the range of 0 to 1 .

Now, we prove the lemma for size $n$ sets. Consider a set $N^{\prime}$ that have $n$ nodes. Let $N^{\prime}=N \cup\{j\}$. Then, we have that:

$$
\begin{aligned}
& \sum_{J^{\prime} \subset 2^{N^{\prime}}-\{i, m\}} a_{J^{\prime}} \Delta T_{i, m}^{k}\left(J^{\prime}\right) \\
= & \sum_{J \subset 2^{N-\{i, m\}}} a_{J \cup\{j\}} \Delta T_{i, m}^{k}(J \cup\{j\})+ \\
& \sum_{J \subset 2^{N-\{i, m\}}} a_{J} \Delta T_{i, m}^{k}(J)
\end{aligned}
$$

$$
=\underbrace{\sum_{J} a_{J \cup\{j\}} \Delta T_{i, m, j}^{k}(J)}_{A}+\underbrace{\sum_{J} a_{J} \Delta T_{i, m, \bar{j}}^{k}(J)}_{B} .
$$

Applying the inequality we got above to Part A and B in Equation 11, we obtain the following.

$$
\begin{aligned}
\left|\sum_{J} a_{J \cup\{j\}} \Delta T_{i, m, j}^{k}(J)\right| \leq & \left|\tau_{m}^{k} \tau_{j}^{k}-\tau_{m}^{k-1} \tau_{j}^{k-1}\right| \\
\left|\sum_{J} a_{J} \Delta T_{i, m, j}^{k}(J)\right| \leq & \mid \tau_{m}^{k}\left(1-\tau_{j}^{k}\right)- \\
& \tau_{m}^{k-1}\left(1-\tau_{j}^{k-1}\right) \mid .
\end{aligned}
$$

Compute the sum of part A and B as follows:

$$
\begin{aligned}
\mid \text { Part } \mathrm{A}+\mathrm{B} \mid \leq & \left|\tau_{m}^{k} \tau_{j}^{k}-\tau_{m}^{k-1} \tau_{j}^{k-1}\right|+ \\
& \left|\tau_{m}^{k}\left(1-\tau_{j}^{k}\right)-\tau_{m}^{k-1}\left(1-\tau_{j}^{k-1}\right)\right| \\
\leq & \left|\tau_{m}^{k}-\tau_{m}^{k-1}\right| . \quad \text { (Lemma 2) }
\end{aligned}
$$

By Equation 14, the first part of Lemma 4 is proved. The second part of Lemma 4 is proved in this way. Let $\tau_{m}^{\prime k}=1-\tau_{m}^{k}$ and $\tau_{m}^{\prime k-1}=1-\tau_{m}^{k-1}$. Putting node $m^{\prime}$ instead of $m$ keeps the number of elements in set $N$ to be the same as before. By the first part of the lemma, we have the following:

$$
\begin{aligned}
\left|\sum_{J \in 2^{N-\left\{i, m^{\prime}\right\}}} a_{J} \Delta T_{i, m^{\prime}}^{k}(J)\right| & \leq\left|\tau_{m}^{\prime k}-\tau_{m}^{\prime k-1}\right| \\
& =\left|1-\tau_{m}^{k}-\left(1-\tau_{m}^{k-1}\right)\right| \\
& =\left|\tau_{m}^{k}-\tau_{m}^{k-1}\right| .
\end{aligned}
$$

Thus, the second part is proved.

The following theorem proves $D^{k}<D^{k-1}$ for all $k>1$ using Lemma 1 to 4.

Theorem 1 (Monotonicity of the Maximum Difference):

With any initial values $p_{i}^{0}$ and any interference function $f_{i}$, each iteration $k$ in our method satisfies the monotonicity of the maximum difference $D^{k}$.

Proof: Let $D_{m}^{k-1}=D^{k-1}$. For any other node $i$ in the node set $N, p_{i}^{k}$ can be expressed by the following:

$$
\begin{aligned}
p_{i}^{k}= & \tau_{m}^{k} \sum_{J \subset 2^{N-\{i, m\}}} f_{r_{i}}(J \cup\{m\}) T_{i, m}^{k}(J)+ \\
& \left(1-\tau_{m}^{k}\right) \sum_{J \subset 2^{N-\{i, m\}}} f_{r_{i}}(J) T_{i, m}^{k}(J)
\end{aligned}
$$

Note that $f_{r_{i}}(J)$ is independent of this process. From Equation $16, D_{i}^{k}$ is given by:

$$
\begin{aligned}
D_{i}^{k}= & \left|p_{i}^{k}-p_{i}^{k-1}\right| \\
= & \mid \sum_{J} f_{r_{i}}(J \cup\{m\}) \tau_{m}^{k} T_{i, m}^{k}(J)+ \\
& \sum_{J} f_{r_{i}}(J)\left(1-\tau_{m}^{k}\right) T_{i, m}^{k}(J)- \\
& \left(\sum_{J} f_{r_{i}}(J \cup\{m\}) \tau_{m}^{k-1} T_{i, m}^{k-1}(J)+\right. \\
& \left.\sum_{J} f_{r_{i}}(J)\left(1-\tau_{m}^{k-1}\right) T_{i, m}^{k-1}(J)\right) \mid
\end{aligned}
$$




$$
\begin{aligned}
= & \mid \sum_{J} f_{r_{i}}(J \cup\{m\}) \Delta T_{i, m}^{k}(J)+ \\
& \sum_{J} f_{r_{i}}(J) \Delta T_{i, \bar{m}}^{k}(J) \mid \\
\leq & \left|\sum_{J} f_{r_{i}}(J \cup\{m\}) \Delta T_{i, m}^{k}(J)\right|+ \\
& \left|\sum_{J} f_{r_{i}}(J) \Delta T_{i, \bar{m}}^{k}(J)\right|
\end{aligned}
$$

By Lemma 4 and 1,

$$
\begin{aligned}
D_{i}^{k} & \leq\left|\tau_{m}^{k}-\tau_{m}^{k-1}\right|+\left|\tau_{m}^{k}-\tau_{m}^{k-1}\right| \\
& <D_{m}^{k-1}=D^{k-1}
\end{aligned}
$$

From Equation 19, we arrive at the inequality for all nodes $i$ except $m$. Let node $n$ have the next largest difference in step $(k-1)$. That is $D_{n}^{k-1}=\max _{i \neq m} D_{i}^{k-1}$. For node $m, p_{m}^{k}$ can be given by:

$$
\begin{aligned}
p_{m}^{k}= & \tau_{n}^{k} \sum_{J \subset 2^{N-\{m, n\}}} f_{r_{m}}(J \cup\{n\}) T_{m, n}^{k}(J)+ \\
& \left(1-\tau_{n}^{k}\right) \sum_{J \subset 2^{N-\{m, n\}}} f_{r_{m}}(J) T_{m, n}^{k}(J)
\end{aligned}
$$

By the same reasoning as in Equation 18 to 19, we can obtain that $D_{m}^{k}<D_{n}^{k-1}$, which is less or equal to $D^{k-1}$. Thus, we have that $D_{m}^{k}<D^{k-1}$ for node $m$.

We have proved Lemma 4 for the sum over all subsets of $2^{N-\{i, m\}}$. However, we can have the same lemma for the sum of some subsets that have up to $t$ elements. That is because we can set $a_{J}=0$ for all $J,|J|>t$. The new lemma follows without proof.

Lemma 5: Consider node set $N$ and nodes $i, m \in N$. Assume that $\left|\tau_{m}^{k}-\tau_{m}^{k-1}\right|$ is greater than or equal to $\left|\tau_{j}^{k}-\tau_{j}^{k-1}\right|$, where $j \in N-\{i, m\}$. Given $t \leq|N-\{i, m\}|$, for any numbers $a_{J},\left|\sum_{J \in 2^{N-\{i, m\}},|J| \leq t} a_{J} \Delta T_{i, m}^{k}(J)\right| \leq\left|\tau_{m}^{k}-\tau_{m}^{k-1}\right|$. In addition, $\left|\sum_{J \in 2^{N-\{i, m\}},|J| \leq t} a_{J} \Delta T_{i, \bar{m}}^{k}(J)\right|$ is less than or equal to $\left|\tau_{m}^{k}-\tau_{m}^{k-1}\right|$.

With Lemma 1 and 5, we can obtain the following corollary using the same reasoning as was used in Theorem 1.

Corollary 1 (Theorem 1 with the Maximum Set Size t):

With any initial values $p_{i}^{0}$ and any interference function $f_{i}$, each iteration $k$ computing Equation 7 instead of 6 in our method also satisfies the monotonicity of the maximum difference $D^{k}$.

In the next section, we compare our analysis with simulations varying $t$.

\section{Simulation AND Verification}

\section{A. Simulation Tool and Modification}

We use Qualnet [16] for our simulations. Qualnet is a commercial version of GloMoSim, which provides a very accurate and realistic simulation environment [8]. For example, Qualnet calculates the power of interference as the sum of all signals other than the one being received. Qualnet also uses the BER based model, which probabilistically decides whether or not each frame is received successfully based on the

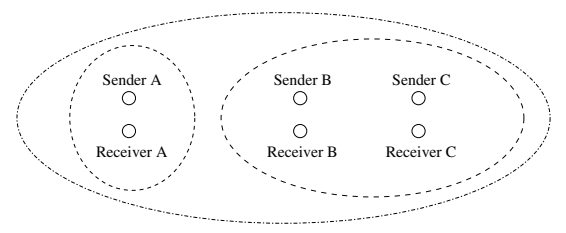

Fig. 2. Fairness problem with EIFS durations in 802.11 .

frame length. ns - 2 [17], probably the most popular network simulator in the open source domain, simply compares the strength of each interference signal with a predetermined signal-to-noise ratio threshold (CPThresh) to determine the packet corruption.

When evaluating our model based on Qualnet, we identified several problems in 802.11 and its simulators. The problems are 1) unfairness from Extended IFS (EIFS), 2) capturing only frames that arrive first, and 3) using backoff timers in nanosecond units. The next three sections explain what the problems that exhibited these behaviors are and how we accounted for and fixed them in our simulations.

\section{B. Fairness and EIFS}

In the 802.11 standard [18], the EIFS must be used by the 802.11 MAC layer (DCF) whenever a frame transmission begins but does not result in the correct reception of a complete MAC frame. If a node is already in its EIFS duration, what should it do if it again receives a corrupted frame? One solution is to start another EIFS interval after the channel is idle, since it may need to wait for a following acknowledgment frame. This approach was implemented in Qualnet and ns -2 .

The approach does avoid conflicts but is not very fair. Figure 2 depicts this issue, using three pairs of senders and receivers that are within the same carrier-sensing and interference range. All three senders can sense signals from one another and all collisions result in packet losses. Their transmission ranges, however, are different. Only node pairs of B and C can communicate. Sender A and Receiver A can talk to each other but can not hear from the other nodes. This usually happens when a sender transmits packets at high rates such as $54 \mathrm{Mbps}$ in 802.11a, where only nodes close to the senders can overhear.

We performed simulations with the same topology in Figure 2 and plotted the results in Figure 3. Running the original Qualnet implementation produced a very unfair bandwidth allocation. The result is denoted by "EIFS On". With the EIFS rule, a sender must go into EIFS mode and wait until either receiving an error-free frame or sensing that the channel is idle for a long time. If Sender C starts its transmission, Sender A does not correctly receive the signal and goes into the EIFS mode. Note that Sender B can receive the frame and may access the channel via 802.11 backoff. Transmission from Sender B also keeps Sender A in the EIFS state but allows Sender $\mathrm{C}$ to access the channel. By repeating this process, Sender A stays in the EIFS state and Sender B and C share 


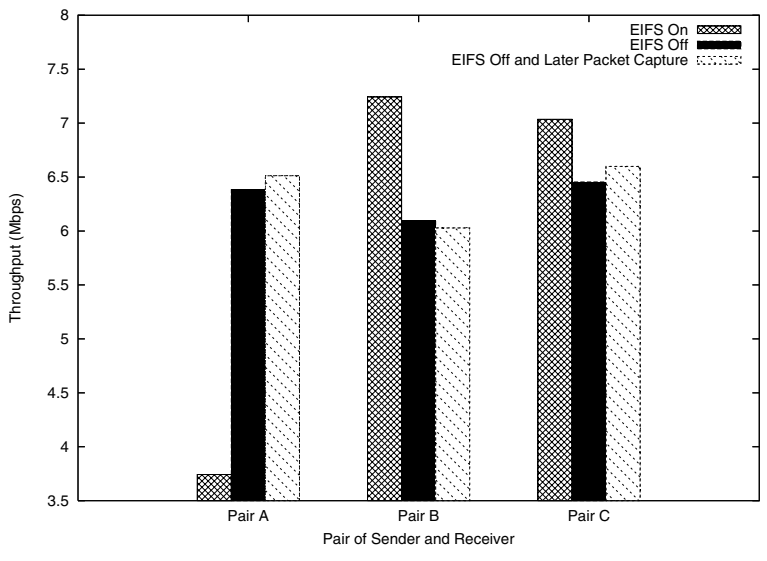

Fig. 3. Throughput results in Qualnet.

TABLE I

Transmission ERror Probability of Node Pairs

\begin{tabular}{c||c|c|c}
\hline & Pair A & Pair B & Pair C \\
\hline \hline EIFS On & 0.06744 & 0.06899 & 0.06661 \\
\hline EIFS Off & 0.09146 & 0.10537 & 0.08706 \\
\hline EIFS Off \& Capture & 0.07785 & 0.10242 & 0.07385 \\
\hline
\end{tabular}

the channel, even though the collision probabilities of three senders are almost the same as shown in Table I.

To address this problem, we introduce the following two approaches. Z. Li et al also present a solution with modification of the 802.11 protocol [19].

1) No EIFS duration: if all nodes are within the same carrier-sensing range, we do not need to use the EIFS duration. Acknowledgments have a higher priority and senders defer their transmissions by sensing the signal. No explicit delay after receiving error frames is required. Moreover, some 802.11 chipsets such as Atheros have a configurable EIFS duration. By setting the EIFS duration to the same as DIFS, we can effectively eliminate the delay.

2) Low speed transmissions of RTS/CTS and acknowledgments: transmitting RTS/CTS and acknowledgments at low speeds increases the communication range and lets neighbor nodes exit the EIFS state. Note that receiving an error-free frame permits nodes to revert to the normal 802.11 backoff process. However, the carrier-sensing range is usually larger than the communication range and even low-speed transmissions may not reach all neighbor nodes. In special cases, the sending of RTS/CTS and acknowledgments at low transmission rates significantly reduces the channel efficiency.

We perform our simulation without the EIFS rule and Assume that all nodes are within the same carrier-sensing range. This is because we do not focus on the fairness problem in this paper and our analysis results may be used to identify reasonable fairness for all nodes. Throughput without the EIFS rule is denoted by "EIFS Off" in Figure 3, which shows

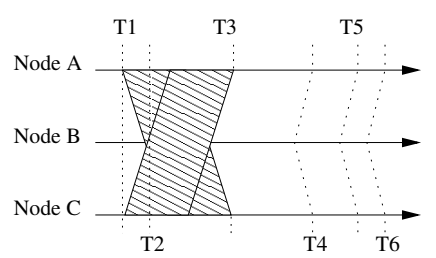

Fig. 4. Time desynchronization in Qualnet. In Qualnet, nodes use backoff timers in unit of microsecond. When sensing a signal in the middle of a time slot, the node immediately pauses its timer without adjusting. It causes clock drift and desynchronization of senders.

fair sharing by three senders. Note that intuitively, sender B must gain lower throughput because its average distance to neighbors is shorter than the other nodes'. Table I shows in clear that more frequent transmission errors occur to sender B not using the EIFS, which matches our intuition.

\section{Correct Physical Layer Capture Model}

Kochut et al point out in their paper [9] that in reality, a variety of 802.11 wireless cards accept frames with stronger signals regardless of whether the frame arrives before or after reception has started. That is, if a stronger signal comes within the physical layer preamble of the frame being received, the stronger frame is instead captured. They show this empirically and demonstrate that neither Qualnet nor ns-2 models this kind of capturing. They provide a patch for ns -2 but not for Qualnet.

We modify Qualnet to accept stronger signals even after starting a frame reception. Simulations in this paper have been done with the modified version and some results are shown and denoted by "EIFS Off \& Capture" in Figure 3 and Table I. The impact of this modification is not very significant for the cases we consider because receivers are much closer to their senders in comparison to the interference sources.

\section{Slot Synchronization}

According to the 802.11 standard, if the medium is busy at any time during a backoff slot, the backoff procedure must be suspended without decreasing the value of the backoff timer. However, in Qualnet, the backoff timer is decreased by propagation delays, which causes time desynchronization.

For example, consider three 802.11 nodes A, B and C as shown in Figure 4. At time $T_{1}$, they are perfectly synchronized. Node A and C starts transmission and node B senses the signals after the propagation delay time, $\delta$. In Qualnet, node B simply pauses its backoff timer at this time. Assuming node B's timer value at time $T_{1}$ is $T$, the timer pauses at time $T_{2}$ with a value of $T-\delta$.

Let node $\mathrm{A}$ and $\mathrm{C}$ choose a new timer value $T$ after the collision. At time $T_{4}$, after a pause due to the previous collision, all nodes resume their backoff procedures. Since node B first determines that the channel is idle, it starts decreasing its backoff timer a time $\delta$ before than the others. That is why we plot the time line $T_{4}$ as an angle bracket. Let $T_{5}=T-\delta$ time after $T_{4}$ and $T_{6}$ be $T$ time after $T_{4}$. Node A 
TABLE II

802.11A OPERATION PARAMETERS

\begin{tabular}{c|c||c|c}
\hline \multicolumn{2}{c||}{ Physical Layer } & \multicolumn{2}{c}{ MAC Layer } \\
\hline \hline Frequency & $5 \mathrm{GHz}$ & MAC Protocol & DCF \\
\hline Path Loss Model & Two Ray & Slot Time & $9 \mu \mathrm{s}$ \\
\hline Shadowing Model & Constant & SIFS Time & $16 \mu \mathrm{s}$ \\
\hline Shadowing Mean & $4 \mathrm{~dB}$ & DIFS Time & $34 \mu \mathrm{s}$ \\
\hline Fading Model & None & $C W_{\min }$ & 15 \\
\hline Temperature & $290 \mathrm{~K}$ & $C W_{\max }$ & 1023 \\
\hline Noise Factor & 7 & MAC Header & 28 bytes \\
\hline Tx Power & $16 \mathrm{dBm}$ & ACK Frame Size & 14 bytes \\
\hline Rx Sensitivity & $-87 \mathrm{~dB}$ & Retry Number & 7 times \\
\hline Antenna & Omnidirectional & Access Mode & Basic \\
\hline Antenna Efficiency & 0.8 & Connection Mode & Ad Hoc \\
\hline Antenna Loss & $0.5 \mathrm{~dB}$ & Routing Protocol & STAR \\
\hline Antenna Height & $1.5 \mathrm{~m}$ & STAR Mode & LORA \\
\hline
\end{tabular}

and $\mathrm{C}$ would try transmission at $T_{6}$, but node $\mathrm{B}$, whose timer value is $T-\delta$, starts earlier at $T_{5}$. The signal reaches nodes A and $\mathrm{C} \delta$ before $T_{6}$ and node $\mathrm{B}$ avoids a collision.

In the 802.11 standard, however, all transmissions from the three nodes must collide since the selected timer values are the same. To guarantee the correct operation, the standard requires node $\mathrm{B}$ reset its timer value to $T$ when sensing a signal at $T_{2}$. This prevents backoff timers from drifting as much as propagation delays.

The time desynchronization in Qualnet allows some nodes to have unreasonably lower collision rates than others. Using the original version of Qualnet, the collision probabilities are reduced to a half of what our analysis expected. For our simulations, we have used our modified version of Qualnet with a decreasing timer as specified in the 802.11 standard.

\section{E. Simulation Parameters}

1) Radio Channel Model: We validate our analysis model and algorithm, using the two-ray path loss model [20]. Multipath fading is not considered. As we mentioned in the previous section. However, other physical layer effect including multipath fading can be take into consideration with interference functions. Table II shows 802.11a physical layer parameters for our simulations.

2) 802.11a Operation Parameters: In our simulation, each node runs 802.11 DCF without RTS/CTS exchanges. The maximum retransmission number is 7 , the same as the 802.11 default value. Each sender and receiver pair is connected in ad hoc mode. We use the STAR (Source Tree Adaptive Routing) routing protocol [21] to prevent the routing protocol from obstructing data transmissions. The protocol operates in LORA mode, where STAR attempts to provide viable, if not necessarily optimal (according to delay metrics) paths to each destination. The message send frequency is set to 100 seconds and packet drops do not trigger routing protocol recovery procedures.

3) CBR Application and Node Placement: Each sender transmits UDP/IP packets to one predetermined receiver using the same CBR source rate. The packet size is 540 bytes

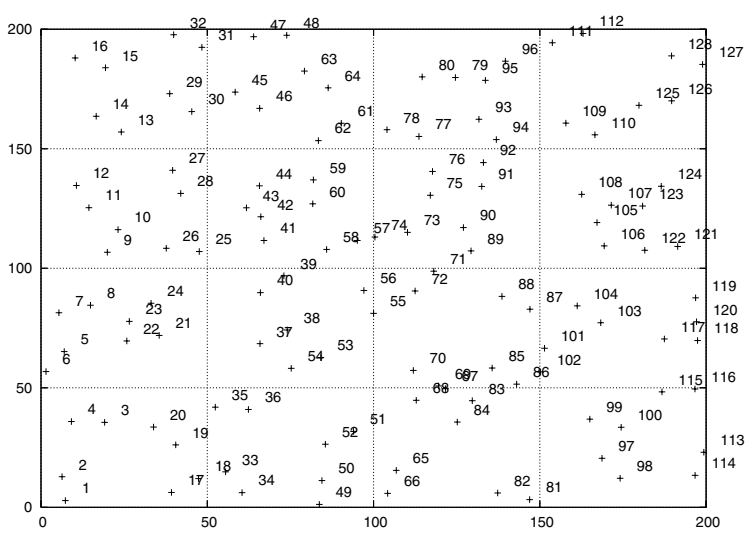

Fig. 5. 64 node pairs placed with random seed 79 .

including UDP and IP header. The CBR application supplies packets to the sender as fast as it can, hence senders always have packets to send in their buffers.

We place pairs of senders and receivers in a $200 \times 200$ square-meter area. The number of sender-receiver pairs is varied from 1 to 64 . Given the number of pairs, we locate pairs at random points, varying the random location for each simulation run. The area is divided into small but equal-sized square spaces and each sender-receiver pair is put in a random square within a space. We arrange senders and receivers from bottom to top and from left to right. Senders with node Id $1+16 n$ are thus located at the bottom. If the number of pairs is less than the number of squares, some squares are empty.

Our node placement emulates typical office and resident environments, where one private access point is installed somewhere in each room and only one client is connected to it a few meters away. The distance between a sender and its receiver is fixed to 10 meters unless specified otherwise. This is close enough so that packets are received at $54 \mathrm{Mbps}$ without any errors if no other transmissions collide. A sample node placement is shown in Figure 5

\section{F. Complexity and Accuracy of Analysis}

To reduce the computation time of our analysis, we have suggested keeping $t$ small. Recall that $t$ in Equation 7 limits the number of interferers; when $t=1$, only one interfering sender is considered to compute the error probability. For $t=2$, all pairs of two concurrent senders are considered. To evaluate the effect of $t$, we perform the analysis with $t$ from 1 to 3 and Figure 6 shows the results. We have repeated our iteration step ten times (i.e., $K$ in Algorithm 1 is set to 10).

Figure 6 plots the results, depicting the ratio of analytical results over simulation results. In Figure 6, analysis considering only one interferer overestimates the average throughputs. This is because a sender has more than one concurrent interferer as the number of senders grows. When considering an increasing number of concurrent interferers, the analysis error reduces and the results from our analysis approach the simulation 


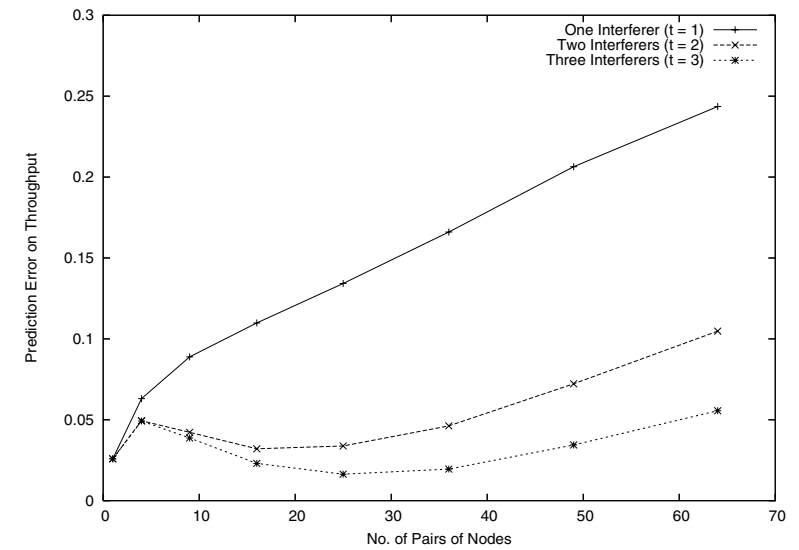

Fig. 6. Analysis results varying the complexity parameter $t$. Analysis error is measured as the throughput difference from the expectation by our analysis over the simulation result. Results from $54 \mathrm{Mbps}$ transmissions are displayed. The results indicate that for larger number of senders, more interferers must be considered.

results. Setting $t=3$, we get reasonable error ratios for 1 to 64 node pairs and the analysis takes $O\left(n^{4}\right)$ time. In the next section, we present analysis results with $t=3$ and $K=10$.

\section{G. Analysis Verification}

Figure 7 shows the cumulative throughputs of 9, 25, 49 and 64 node pairs with 10 different random topologies. We do not present results for 1, 4, 16 and 36 node pairs due to space limitations. Figures from 10 to 13, however, include all results with all numbers of pairs.

In Figure 7, the first 10 black bars represent the throughputs of 9 node pairs with 10 topologies. The next 10 black bars are for 25 node pairs and so on. White bars next to black bars show the expected throughputs from our analysis with the same topology and the same number of node pairs.

Even though we have omitted some results, the accuracy of our analysis is clear. For $36 \mathrm{Mbps}$ transmissions, the analysis on small numbers of node pairs achieves higher accuracy, but the ratio of analysis to simulation results are limited at around 12 percent. For $54 \mathrm{Mbps}$, the accuracy is more enhanced and the errors to simulation results are less than 6 percent.

To verify our analysis on throughput and error probability for each individual node, we pick the worst error cases in Figure 7. One is 64 node pairs (i.e., 128 nodes) at $36 \mathrm{Mbps}$ with random seed 79 and the other is 64 node pairs at 54 Mbps with seed 73. Node placements with random seed 79 is depicted in Figure 5.

Figure 8 and 9 depict individual throughputs and error probabilities from the analysis and simulations. In the graphs, the $x$ axis contains the node pair ID, and the $y$ axis shows throughput and error probability of transmissions from the sender in the pair. The graph in Figure 8 plots both of simulation and analysis results at 36 and 54 Mbps. Node throughput varies in a wide range even though nodes are in the same carrier-sensing range. Our analysis produces small expectation errors except for low-throughput nodes. In the

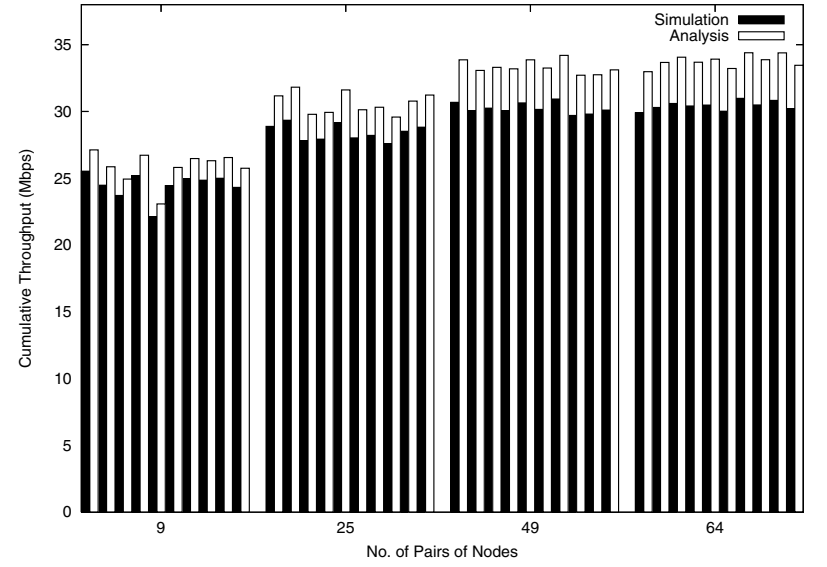

(a) Throughputs of $36 \mathrm{Mbps}$ transmissions

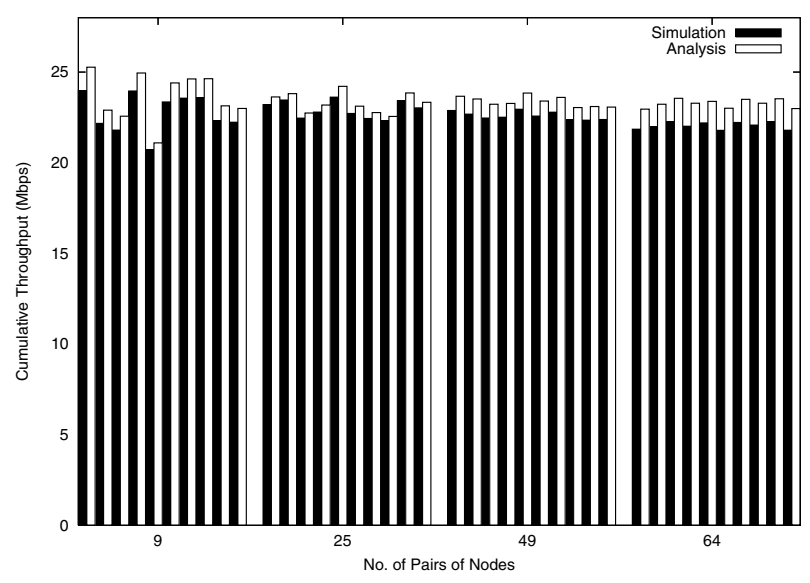

(b) Throughputs of 54 Mbps transmissions

Fig. 7. Throughputs of $36 \mathrm{Mbps}$ transmissions. Given the number of senderreceiver pairs, 10 simulations have been performed with different random seeds. Black bars present simulation results. Expectation from analysis is shown by white bars. The analysis error to simulation result slightly increases as the number of pairs grows. The maximum error for $36 \mathrm{Mbps}$ transmission is $11.5313 \%$ when nodes are 64 pairs and the random seed is 79 . For 54 Mbps, the maximum error is $5.8231 \%$ with 64 pairs and random seed 73 .

graph in Figure 9, at $36 \mathrm{Mbps}$, the maximum analysis error is $12.2879 \%$ with pair Id 31 . For nodes at $54 \mathrm{Mbps}$, the maximum error is only $4.2429 \%$ (pair Id 2). In Bianchi's model, all nodes must have the same error probability, which is 0.63247 in this case.

The graphs shows the accuracy of our analysis without large variances for individual node pairs. For pair throughput, the analysis error is small except for low-throughput nodes. A more statistically significant result is the accuracy of expected error probabilities. Even in the two worst cases, the error probabilities from our analysis are almost the same as in the simulation results. This incredibly accurate prediction clearly proves our iteration method computes highly accurate error 


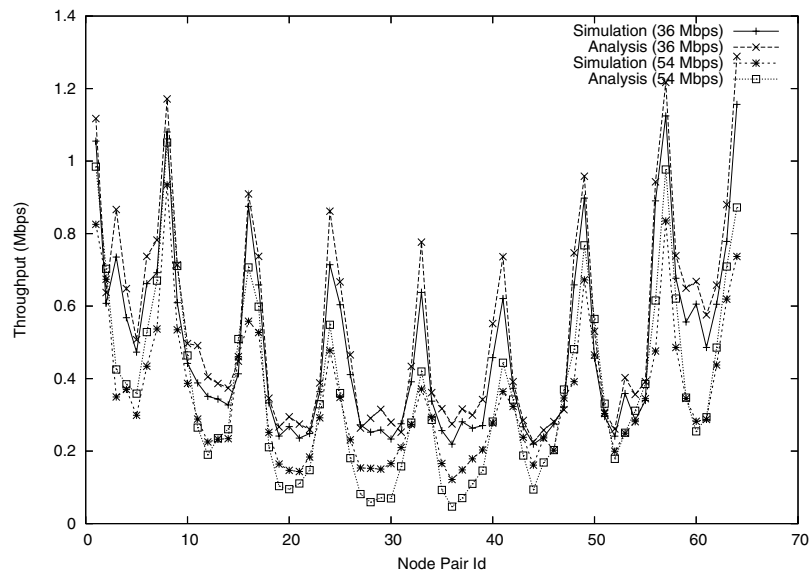

Fig. 8. Throughputs of individual nodes.

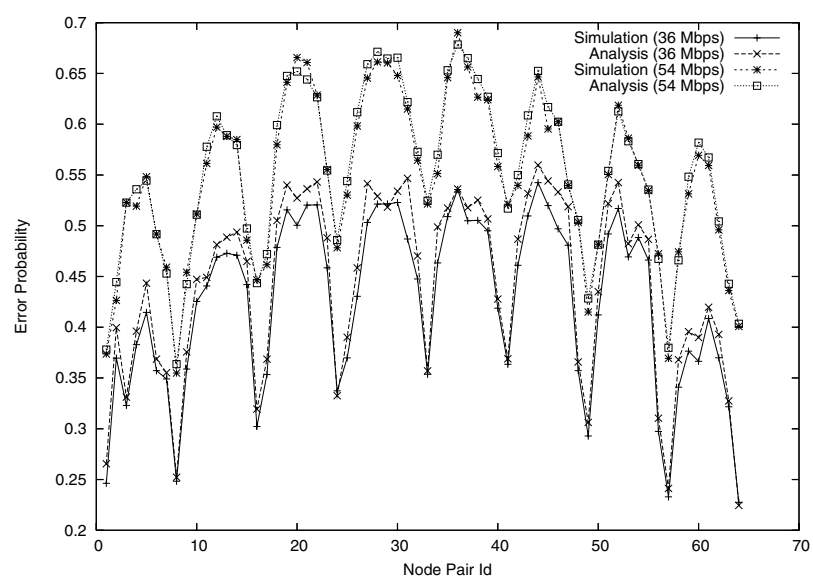

Fig. 9. Error probabilities of individual nodes.

probabilities in reasonable running time $O\left(n^{4}\right)$. The accuracy of our method for all simulation results is further supported by the histograms in Figures 10 through 13.

To plot error histograms, we compute the prediction error with respect to the throughput or error probability values observed for all node pairs in all simulations. Since we have performed simulations with 10 different topologies, varying the number of pairs from 1 to 64 , the total number of node pairs is 2,040 . The following error histograms show individual frequencies for error ranges in 5 percent increments.

For throughput of each pair, the prediction error is within 20 percent in 94.42 percent of node pairs at $36 \mathrm{Mbps}$ and 83.38 percent at $54 \mathrm{Mbps}$. For error probabilities, our method predicts within a $10 \%$ error for more than $98 \%$ of then nodes. These statistical results show our iteration method achieves extreme accuracy with only a few iterations.

We also perform our analysis in cases with random distance. Figure 12 and 13 show these results. We place receivers at random distances in the range of 5 to 10 meters from their senders. Each pairs are placed as in the previous simulations. As shown in Figure 13, our iteration method can predict

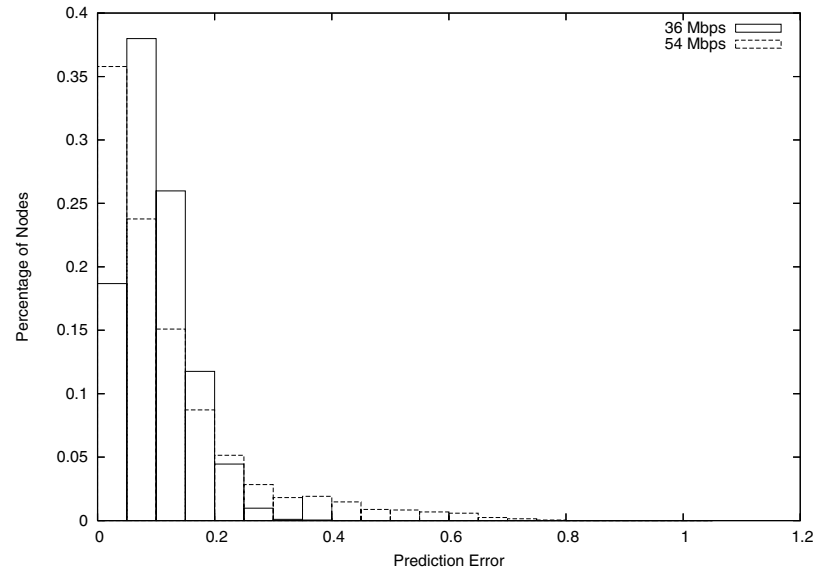

Fig. 10. Error histogram on individual throughputs at 36 and 54 Mbps. The prediction error is computed by the ratio of the difference between the expectation from our analysis and the results from simulations to the simulation results. The average of prediction errors in total 4,080 cases (including all of 36 and $54-\mathrm{Mbps}$ transmission results) is $10.8463 \%$.

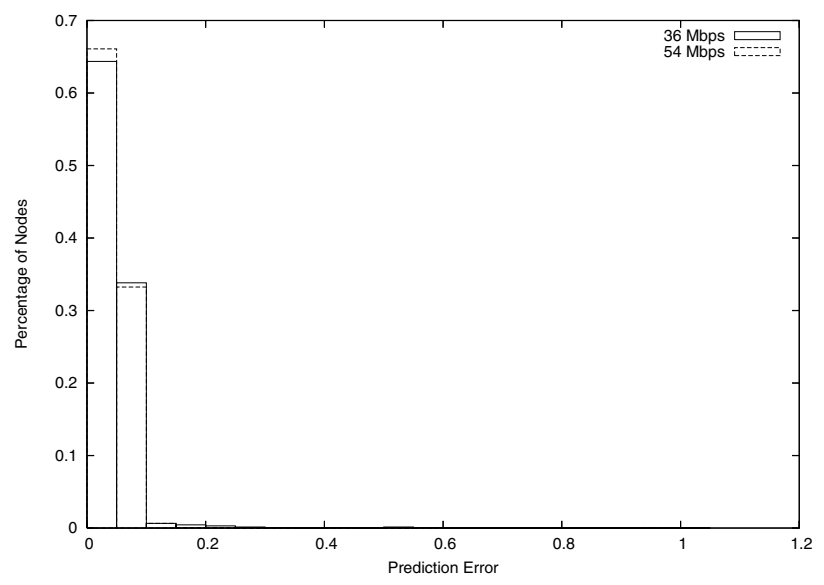

Fig. 11. Error histogram on transmission error probabilities at 36 and 54 Mbps. Our iteration method achieves highly accurate prediction. Our method predicts within $10 \%$ error for more than $98 \%$ of nodes.

accurate transmission errors. Our method still shows high accuracy even though the average prediction error is slightly higher $(4.6683 \%)$ than fixed-distance cases $(4.1884 \%)$. For node throughputs, the prediction error is larger than before. That is because the number of low-throughput nodes increases in random-distance cases, which enlarges the prediction error with even small differences.

\section{Vi. Application: Delay Enhancement}

\section{A. Overview}

As an application, we present a rate assignment algorithm, which uses our analysis method to compute the average delivery delay of all nodes. Consider an area that has two types of rooms: one type of rooms are big and the other type is small. In small rooms, the sender-receiver distance is 1.25 meters on average while the average distance is 5 in large rooms. 


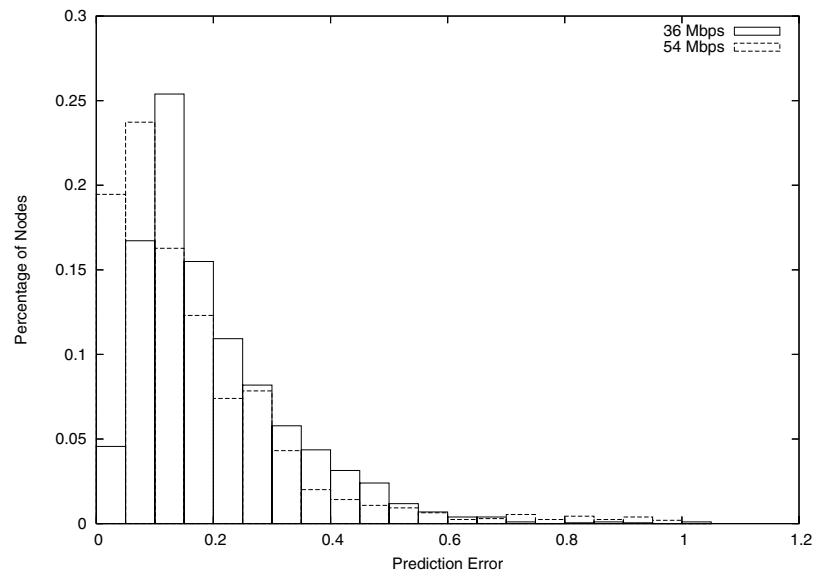

Fig. 12. Error histogram on individual throughputs. We set the distance between a sender and a receiver randomly in the range of 5 to 10 meters. The prediction error becomes a little bit higher than fixed-distance cases. However, the difference between the expectation and the simulation results is only 0.134512 Mbps on average.

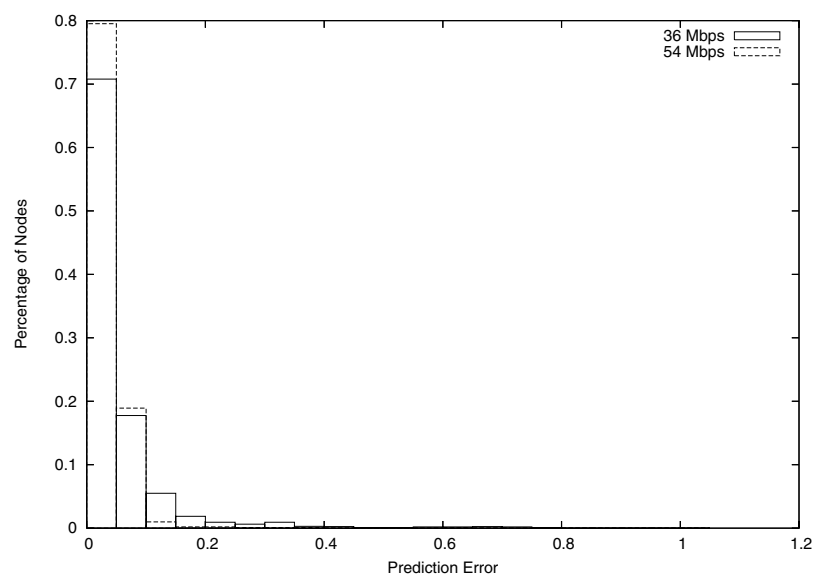

Fig. 13. Error histogram on error probabilities with random distance.

In this example, frames transmitted at $54 \mathrm{Mbps}$ in small rooms can be captured at receivers. For senders in large rooms, however, transmitted packets are frequently lost and the average delivery delays are high. If a lower transmission rate is assigned to senders located in large rooms, it increases capture probabilities of frames and reduces delivery delays. However, the senders occupy the channel longer which increases delays from senders in small rooms. Hence, to determine the average delay of the entire network, we need to measure the tradeoff of low rate assignments.

Given two available rates $r_{l o w}$ and $r_{\text {high }}$, Algorithm 2 shows how to assign transmission rates to each node. The algorithm uses a greedy approach. The idea is to pick a node, assign the node the lower rate, $r_{l o w}$, and perform our analysis to evaluate the average delay. If the average has been improved, the assignment is accepted; otherwise, the assignment is canceled and the node's rate reverts to its original value, $r_{\text {high }}$. Intuitively, nodes with large error probabilities should

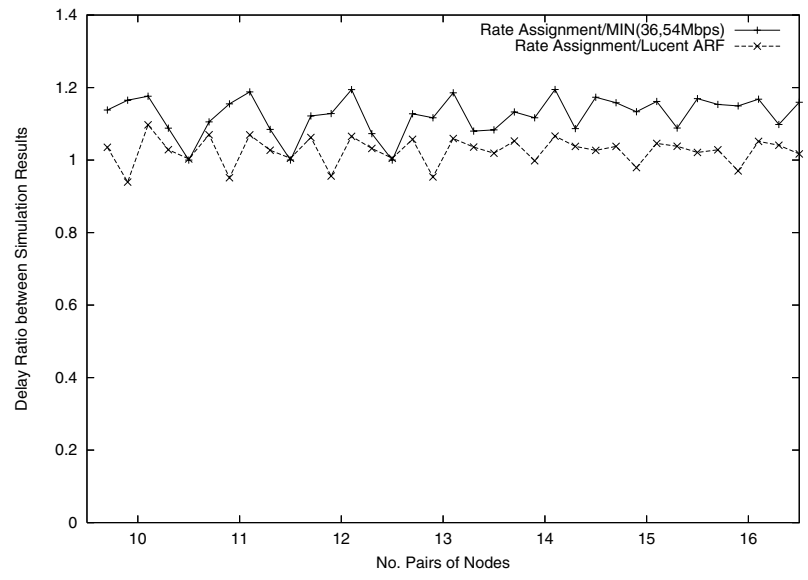

Fig. 14. Delay enhancement in 802.11a.

be considered first for lower rate assignment and our algorithm visits nodes in that order. Since our analysis procedures take $O\left(n^{t}\right)$ time, the total time of Algorithm 2 is $O\left(n^{t+1}\right)$.

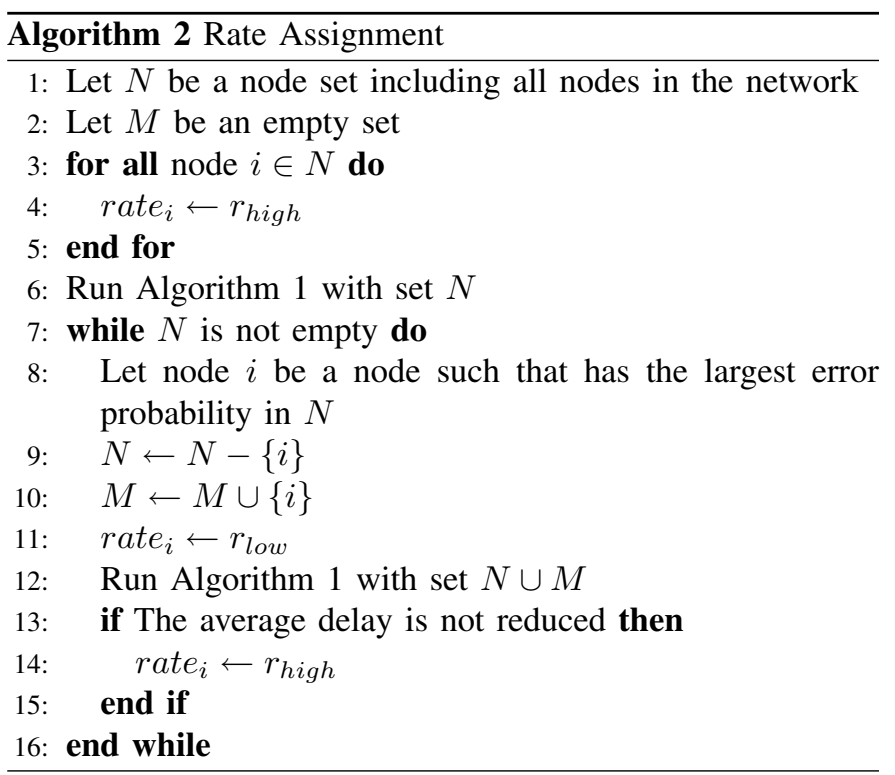

\section{B. Simulation Results}

Figure 14 shows simulation results. We have varied the number of node pairs from 10 to 16 and positioned a sender/receiver pair with distances of either 1.25 or 5 meters on average. The probability that a pair is in a small room is 0.5. All other simulation parameters are set as described in Section V. For each set of pairs, we have performed simulations with 5 random topologies and three different rate assignment schemes. The assignment schemes are 1) all nodes transmit at $36 \mathrm{Mbps}$, 2) at $54 \mathrm{Mbps}$, or 3) at either $36 \mathrm{Mbps}$ or $54 \mathrm{Mbps}$ that is assigned to each node by Algorithm 2.

In Figure 14, the graph denoted by "Rate Assignment/MIN" shows the minimum average delay of rate assignment schemes 1 and 2 with respect to the average delay by our assignment 
algorithm. For 10 to 16 node pairs, our rate assignment reduces average delays by 11 percent on average. Moreover, in all cases, our algorithm always achieves average delay less than or at least equal to the minimums of schemes 1 and 2. We also compares our assignment algorithm with Lucent AutoRate Fallback (ARF) [22]. In Figure 14, our algorithm's resulting delays are up to 10 percent smaller than what is achieved using the ARF scheme. That is because traditional rate adaptation schemes automatically choose the appropriate modulation scheme (data rate) to match the channel conditions.

If packet losses are mainly due to collisions, however, such adaptation is impossible. Because 802.11 MAC operations cause collisions to occur following a random pattern, and ARF rate adaptation rather reduces the channel efficiency. In addition, rate adaptation schemes only consider the local channel conditions at a pair of a sender and a receiver. Because of the tradeoff of rate changing we mentioned earlier, the schemes may not obtain optimal average delay times.

\section{CONCLUSION}

In this paper, we have presented a general analytical model to predict error probability and throughput of packet transmissions with multiple sender-receiver pairs. We have focused on wireless networks in a small area, where senders and receivers communicate at short distances and potential interferers are far away. Assuming high packet capture rates, we have provided an equation computing the total throughput based on error probability and channel access rate.

To compute the error and access rates from given interference functions, we have developed an iterative method. Compared to previous works, the major improvement of our method is to consider the cumulative strength of interference signals. This allows analyzing packet reception under any interference from the behavior of neighbors. We also proved that our iterative method converges.

Detailed simulation results in Qualnet are performed to verify the accuracy of our model. In particular, our method predicts transmission failure probabilities with extremely small errors. The prediction errors are reduced as more concurrent interferers are considered, which demonstrates the importance of considering multiple interferers and the cumulative signal strength.

We have discussed the EIFS problem and other problems in Qualnet. We have explained the problems in detail and how to fix them. Last, as an application of our analysis, we present a rate assignment algorithm to reduce average delay. Our low-complexity rate assignment algorithm can reduce the average delays in various topologies by 11 percent.

\section{REFERENCES}

[1] M. Soroushnejad and E. Geraniotis, "Probability of capture and rejection of primary multiple access interference in spread spectrum networks," in IEEE Transactions on Communications, vol. 39, no. 6, 1991, pp. 986-994.

[2] J. Y. Zhao and O. C. Ugweje, "Analysis of capture probability performance techniques for wireless lan," in IEEE 55th Vehicular Technology Conference, vol. 3, 2002, pp. 1190 - 1194.
[3] J. H. Kim and J. K. Lee, "Capture effects of wireless CSMA/CA protocols in rayleigh and shadow fading channels," in IEEE Transactions on Vehicular Technology, vol. 48, no. 4, 1999, pp. 1277 - 1286.

[4] Z. Hadzi-Velkov and B. Spasenovski, "Capture effect in IEEE 802.11 basic service area under influence of rayleigh fading and near/far effect," in The 13th IEEE International Symposium on Personal, Indoor and Mobile Radio Communications, vol. 1, 2002, pp. 172 - 176.

[5] — "On the capacity of IEEE 802.11 DCF with capture in multipathfaded channels," in International Journal of Wireless Information Networks, vol. 9, no. 3, 2002, pp. 191-199.

[6] M. M. Carvalho and J. J. Garcia-Luna-Aceves, "A scalable model for channel access protocols in multihop ad hoc networks," in MobiCom '04: Proceedings of the 10th annual international conference on Mobile computing and networking. ACM Press, 2004, pp. 330-344.

[7] Y. Wang and J. J. Garcia-Luna-Aceves, "Performance of collision avoidance protocols in single-channel ad hoc networks,' in ICNP '02: Proceedings of the 10th IEEE International Conference on Network Protocols. Washington, DC, USA: IEEE Computer Society, 2002, pp. 68-77.

[8] M. Takai, J. Martin, and R. Bagrodia, "Effects of wireless physical layer modeling in mobile ad hoc networks," in MobiHoc '01: Proceedings of the 2nd ACM international symposium on Mobile ad hoc networking \& computing. New York, NY, USA: ACM Press, 2001, pp. 87-94.

[9] A. Kochut, A. Vasan, A. U. Shankar, and A. Agrawala, "Sniffing out the correct physical layer capture model in $802.11 \mathrm{~b}$," in ICNP 2004: 12th International Conference on Network Protocols, 2004.

[10] C. Ware, J. Judge, J. Chicharo, and E. Dutkiewicz, "Unfairness and capture behaviour in 802.11 adhoc networks," in IEEE International Conference on Communications, vol. 1, 2000, pp. 159 - 163.

[11] G. Bianchi, "Performance analysis of the IEEE 802.11 distributed coordination function," IEEE Journal on Selected Areas in Communications, vol. 18, no. 3, 2000.

[12] Y. C. Tay and K. C. Chua, "A capacity analysis for the IEEE 802.11 mac protocol," Wirel. Netw., vol. 7, no. 2, pp. 159-171, 2001.

[13] H. S. Chhaya and S. Gupta, "Performance modeling of asynchronous data transfer methods of IEEE 802.11 MAC protocol," Wirel. Netw. vol. 3, no. 3, pp. 217-234, 1997.

[14] H. Pan, S. Sato, and K. Kobayashi, "On the throughput of an IEEE 802.11a wireless LAN system with terminals under heterogeneous radio conditions," in Proceedings of the 18th International Teletraffic Congress (ITC-18), August 2003.

[15] D. N. C. Tse and S. V. Hanly, "Linear multiuser receivers: effective interference, effective bandwidth and user capacity," in IEEE Transactions on Information Theory, vol. 45, no. 2, 1999, pp. 641 - 657.

[16] Scalable Network Technologies, "Introduction to QualNet," Qualnet User's Manual, 2004.

[17] "The Network Simulator - ns-2, v2.28," 2005. [Online]. Available: http://www.isi.edu/nsnam/ns

[18] "Wireless LAN medium access control(MAC) and physical layer (PHY) specifications," IEEE Standard, 1999.

[19] Z. Li, S. Nandi, and A. K. Gupta, "Improving fairness in IEEE 802.11 using enhanced carrier sensing," IEEE Proceedings-Communications, vol. 151, no. 5, pp. 467-472, October 2004.

[20] T. Rappaport, Wireless Communications: Principles and Practice. Prentice Hall, 1996.

[21] J. J. Garcia-Luna-Aceves and M. Spohn, "Source-tree routing in wireless networks," in Proceedings of the 7th International Conference on Network Protocols (IEEE ICNP 99), October 1999, Toronto, Candada. IEEE, October 1999, pp. 273-282.

[22] A. Kamerman and L. Monteban, "WaveLAN-II: A high-performance wireless LAN for the unlicensed band," Bell Labs Technical Journal, pp. 118-133, 1997. 